\title{
Research Article \\ Hamstring tightness causing low back pain among college going students - a cross-sectional study
}

Kamalakannan, M. ${ }^{1}$, Hemamalini P. ${ }^{2}$, Divya T. ${ }^{2}$

${ }^{1}$ Assistant Professor, ${ }^{2}$ Physiotherapy Undergraduate, Saveetha College of Physiotherapy, SIMATS Chennai, Tamil Nadu, India

(Received: December $2019 \quad$ Revised: September $2020 \quad$ Accepted: November 2020)

Corresponding author: Kamalakannan M. Email: kamal1712@gmail.com

\begin{abstract}
Introduction and aim: Posterior thigh muscles are also called the Hamstring group of muscles. Its major action is knee flexion and minor action in hip extensor. It is one of the two joint muscles that involve the hip joint and knee joint. When these muscles are shortened or become tight the movement in the hip and knee is affected or reduced. This shortened muscle causes a posterior pelvic tilt that leads to a flat back. Prolonged tightness causes low back pain. Tightness is mainly due to inadequate physical activity results in stiffness, osteoarthritis, and osteoporosis. The aim of this study is to find whether tight hamstring causes low back pain in college-going students.

Materials and methods: The method used to measure the hamstring length in this study is the Active Knee Extension Test. The knee angle is measure using universal goniometry. The knee angle should be between 80-90 degrees. If the range is below 80 degrees, then that subject has a tight hamstring.

Results: Mean hamstring tightness in males is calculated and found that the left is more affected than the right. The mean hamstring tightness in the female is calculated and found that the right side is more affected than the left side. Both males and females having tightness at the range of 30-40 degrees are more.

Conclusion: This study shows that most of the colleges going students have knee range below 80 degrees. And investigation reveals that $2 / 4^{\text {th }}$ of the total experienced low back pain in their life. This pain experience is present in all age group that comes under this study. Some students who involve in sports activities have tight hamstring. But some do not have because of stretching muscles before and after the sports activity. By this, it is concluded that prevalence is more among college-going, students.
\end{abstract}

Keywords: Hamstring tightness; low back pain; goniometry, SLR

\section{INTRODUCTION}

$\mathrm{P}$ osterior thigh muscles are called hamstring group muscles. They are semitendinosus, semimembranosus, and biceps femoris long and short head. All together flex the knee but the short head of biceps femoris alone extends the hip (1). Hamstring muscles originate from the ischial tuberosity of the pelvis and posterior femur and inserts on the proximal lateral tibia. It is one of the two joint muscles. Hamstring muscles act as strong flexors of the knee and weak extensors of the hip (2). The hamstring is a variable length. Some person cannot touch their fingers while standing as their hamstring muscles are rather short or tight. The sciatic nerve supplies the hamstring group of muscles. The length of the hamstring cannot be directly measured, this measurement is done in the angular measurement of unilateral hip flexion with the knee extended (3). A universal goniometer was used to measure the angle of the knee for hamstring length measurements while the hip was maintained at 90 degrees of flexion (4). It was impossible to conclude whether individuals with LBP have impaired hamstring flexibility and stiffness. Hamstring tightness causes chronic low back pain. It may get worse if left untreated $(5,6)$. On this basis, this study is to investigate the relationship between hamstring flexibility and low back pain. Hamstring muscle extensibility and low back pain are closely related and have a correlation with age and gender. Hamstring tightness increases with age and affects between 40-49 and mostly males are affected hamstring measurement using straight leg raise test is less accurate because of the rotation of pelvis that occurs during the test $(7,8)$. The Active knee extension test is the best method to rule out the extensibility range of the hamstring muscle with the hip stabilized at 90 degrees and with accurate instrument placement. This method gives reliable measurements about hamstring extensibility and length with a 90/90 position in supine. The amount of extension was measured using both an electrogoniometer and a standard goniometer. From this finding, it can be stated whether mechanical low back pain is caused by hamstring tightness $(9,10)$.

The tight hamstring can cause a posterior tilt of the pelvis which causes back pain. Low back pain and gait abnormality are commonly linked with movement 
dysfunction at the lumbar spine, pelvis, and lower limbs. If hamstrings are tight, then blood supply will be squeezed out of them. Therefore, muscle is working with less capacity to do work, which in turn causes low back pain. Hamstring tightness leads to a high risk of recurrent injury and reduces the performance and coordination of athletes. So as to prevent such risk factors for athletes and college-going students this study will be an alarming sign. Because college students often adopt a sitting posture for a long period of time. This causes the hamstring to be in a contracted state for a long time and goes for tightness. Tight hamstring increases stress on low back and aggravates the pain $(11,12)$. Previous history and early onset of low back pain are associated with chronic low back pain in adults, implying that prevention in adolescence may have a positive impact in adulthood. Hence this study was undertaken as there is a lack of literature on the prevalence and severity of hamstring tightness hence purpose of this study was to find hamstring tightness among college-going students. This is just like the screening process.

\section{MATERIALS AND METHODOLOGY}

A cross-sectional study was conducted at Saveetha College of Physiotherapy, Thandalam. Ethical clearance was obtained from the institution. 250 college students were assessed in this study. Informed consent was obtained, Inclusion criteria: The age group of 18-21 years. Exclusion criteria: Subject with any past hamstring injury within the last 2 years, any recent hamstring surgery.

The active knee extension (AKE) test is used to check the range of motion at the knee joint and assess the hamstring tightness. This study was done mainly on college-going students. The procedure is first explained to the subjects and assessed their hamstring length by active knee extension test. For this test, the subjects were assessed on a couch in the supine position with both the lower extremities extended and in a fully relaxed position. Both anterior superior iliac spines are aligned straight. The lower extremity which is not being measured is kept fixed on the couch. The subjects were asked to flex the measuring hip to 90 degrees and extend the knee as much as they can. With this position, the knee angle is measured using the Universal Goniometer in marked joint axis as lateral epicondyle of the femur, and with the fixed arm along the long axis of the femur and the movable arm along the long axis of the tibia. The Active knee extension measurements were defined as the degree of knee extension from the knee at 90 degrees in relation to the hip at 90degree. On each side, measurements are done twice or thrice, and the mean angle is taken which was used for the analysis. There is an association of hamstring tightness and range of Motion in Anterior pelvic tilt, lumbar motion, and trunk.

\section{Statistical analysis}

The collected data were tabulated below. Statistical analysis was done on a blinded, intention to treat basis using SPSS software (version 18.0). $\mathrm{P}<0.05$ was set for all analyses and the significance level was set to $\alpha=$ 0.05 .

Table 1: Hamstring tightness measurement

\begin{tabular}{|c|c|c|c|c|}
\hline \multirow{2}{*}{$\begin{array}{c}\text { Range in } \\
\text { degrees }\end{array}$} & \multicolumn{2}{|c|}{ Male } & \multicolumn{2}{c|}{ Female } \\
\cline { 2 - 5 } & Right & Left & Right & Left \\
\hline $20-30$ & 23 & 18 & 29 & 24 \\
\hline $30-40$ & 57 & 66 & 52 & 32 \\
\hline $40-50$ & 26 & 33 & 21 & 37 \\
\hline $50-60$ & 29 & 23 & 12 & 18 \\
\hline
\end{tabular}

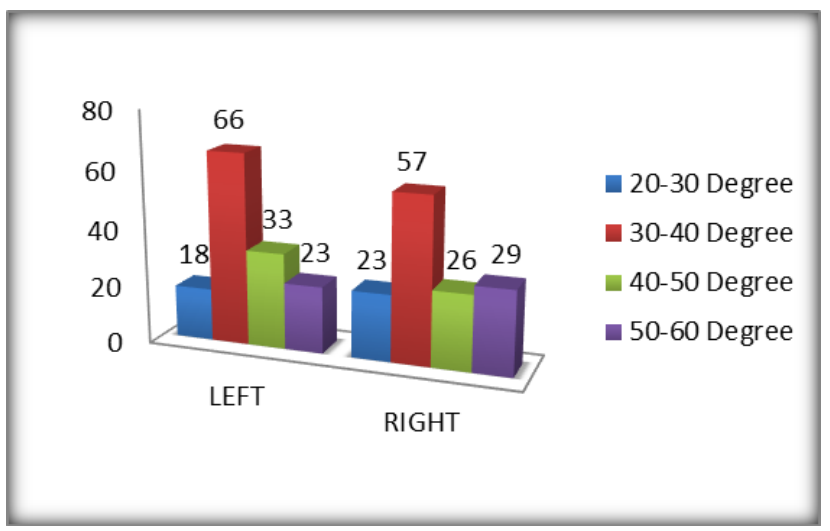

Fig. 1: Hamstring tightness - male

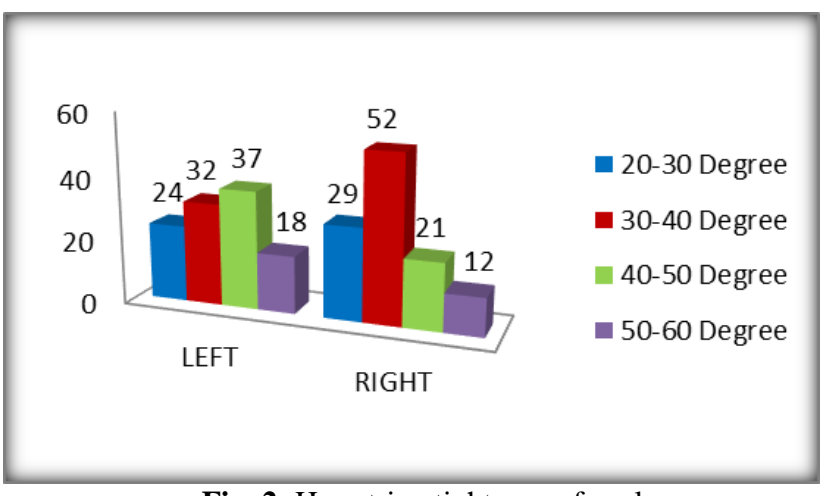

Fig. 2: Hamstring tightness - female

\section{RESULTS}

In this study, there were 250 college-going students, aged between 18-21years were included. With the above-collected data and calculated degree of hamstring tightness, we obtained the mean value. Mean hamstring tightness in males is calculated and found that the left is more affected than the right. The mean hamstring tightness in the female is calculated and found that the right side is more affected than the left side. Both male and female having tightness at the range of 30-40 degree is more.

\section{DISCUSSION}


This will be an alarming sign for the students to prevent upcoming low back problems due to hamstring tightness. This study includes all normal individuals except those with low back pain and lumbar pain for the past 2 years. Lack of mobility caused by tightness or shortness of the ligaments and soft tissue causes hamstring tightness $(13,14)$.

The tight hamstring is a frequent sign in Scheurmann khyphosis after surgery and also gave preoperative assessment for the lumbar pelvic range of motion. There is a direct relationship between tight hamstring and low back pain (15). A study measured the extent of hamstring using electro goniometry and conventional goniometry and resulted that the more the tightness the higher the severity of low back pain. Tightness or shortening of hamstring causes posterior tilt of the pelvis which in turn increases the lumbar lordosis results in severe lower back pain (16).

Hamstring tightness contributed by neural structures causing severe pain in the posterior thigh, knee, leg, and also in the cervical and thoracic region, which was relieved by cervical extension and reduces the discomfort. Passive knee angle was measured with a standard force of $68.7 \mathrm{~N}(7 \mathrm{~kg})$ applied proximal to the lateral malleolus. Hamstring tightness in turn causes patella femoral pain. Pain present in the front of the knee and around the patella, this may be due to vigorous use in athletics or training. Tight hamstring Pull on the hip bone causing a slight rotation which affects the curvature of the back leads to back pain and lower back tightness and increases the incidence of a hamstring strain. From the above discussion, it is clear that hamstring tightness is caused because of the overuse of muscle on college-going student's age 1821 years (17). Tightness occurs in students who are not involved in any physical activities. Their habit of sitting with a flexed knee for the whole day cause the hamstring muscles to shorten which causes the pelvic bone to tilt posterior. The posterior tilt increases the lumbar lordosis causing back muscle strain leads to mechanical low back pain. Low back pain can be regular hamstring stretch and physical activities and often changing the Posture and walking. IA stokes explain that hamstring muscle can restrict hip flexion in standing. An individual with a short or tight hamstring has an abnormal pelvic tilt in seating Posture with greater flattening or reversal of the lumbar lordosis. The range of hip flexion is measured using a toe-touch test. This test is done in standing and sitting hamstring tightness is a lack of ROM with the continuous feeling of restriction in the posterior thigh causing dysfunctional or restricted movement of the Hip flexion $(18,19)$. Hamstring stiffness could be a possible contributing factor to the low back pelvic rhythm. Movement restrictions lead to compensatory movement patterns of the lumbar spine and increase stress on spinal soft tissues and increase the risk of low back pain.

\section{CONCLUSION}

This study shows that most of the college-going students have knee range below 80degree. And investigation reveals that $2 / 4$ th of the total experienced low back pain in their life. This pain experience is present in all age group that comes under this study. Some students who involve in sports activity and use their hip and knee more like football players and athletes etc. have tight hamstring. But some do not have because of stretching muscles before and after the sports activity. By this, we conclude that prevalence is more among college-going, students. This is mainly due to lifestyle adaptation.

\section{CONFLICT OF INTEREST}

There is no conflict of interest from other authors.

\section{REFERENCES}

1. Akinpelu, A. O., Bakare., and B. A., Influence of age on hamstring tightness in apparently healthy Nigerians. Journal of Nigeria Society of Physiotherapy, 2009; 15(2): 35-41.

2. Gajdosik, R. L., comparison of four clinical tests for accessing hamstring muscle length. 1993; 18(5): 614-618.

3. Bellew, S., H. Ford., and Shere, E. The relationship between hamstring flexibility and pelvic rotation around the hip during forward bending. Plymouth Stud J Health Social Work, 2010; 2(2): 19-29.

4. Nikolaidis, P. T. Age-related differences of hamstring flexibility in male soccer players. Baltic journal of health and physical activity. 2012; 4(2): 110-115.

5. Aderone. O., Bakare, U. Influence of age on hamstring tightness in apparently healthy Nigerians. 2005; 15(2): 35-42.

6. Bohannon, R. W. Cinematographic analysis of the passive straight leg raising test for hamstring muscle length, Physical therapy. 1982; 61(1): 1269-1274.

7. Gajdosik, R. L., Lusin, G. Hamstring Muscles tightness. Reliability of an active knee extension test. Physical therapy. 1983; 63: 1083-1090.

8. Radwan, A., Bigney, K. A., Buonomo, H. N., Jarmak, M. W., Moats, S. M., Ross, J. K., et al. Evaluation of intra-subject difference in hamstring flexibility in patients with low back pain: An exploratory study. Journal of back and musculoskeletal rehabilitation. 2015 Jan 1; 28(1): 61-66.

9. Falavigna, A., Teles, A. R., Mazzocchin, T., de Braga, G. L., Kleber, F. D., Barreto, F., et al. Increased prevalence of low back pain among physiotherapy students compared to medical students. European Spine Journal. 2011 Mar 1; 20(3): 500505.

10. Garber CE, Blissmer B, Deschenes MR, Franklin BA, Lamonte MJ, Lee IM, et al. American College of Sports Medicine position stand. Quantity and quality of exercise for developing and maintaining cardiorespiratory, musculoskeletal, and neuromotor fitness in apparently healthy adults: guidance for prescribing exercise. Medicine and science in sports and exercise. $2011 \mathrm{Jul}$;43(7):1334.

11. Bhagyashree, K. Koli, Deepak, B., prevalence and severity of hamstring tightness among college students: A cross-sectional study. 2018;4(2):65-68. 
12. Kamalakannan, M. Efficacy of Proprioception Training for Tibiofemoral Arthritis in relation with Pain and Functional Disability. Research Journal of Pharmacy and Technology 2019 12(3): 995-998

13. Luca. D., Petera.M.D., Sylvia.,Davis., Effect of Hamstring and psoas lengthening on pelvic tilt in patients with spastic Diplegic cerebral palsy.1998; 18(6): 712-718.

14. Kate, E.K., Nicholas, L., The active knee extension test and Slump test in subject with perceived hamstring tightness. 2005; 8(3): 89-97.

15. Kenny, G., Francis, F., Heiko, L., and Gregoire, P., Millet. Passive knee Extension Test to Measure Hamstring Tightness: Influence of Gravity correction. 2012; 21(3) .231-234.

16. Kamalakannan, M., J. Boaz, and M. Koushik Kannan. "Influence of Ankle Mobilization and Active Stretching on Low Back Pain in Pronated Foot Subjects." Research Journal of Pharmacy and Technology 13, no. 3 (2020): 1142-1146.

17. I.A.Stokes., Joan, M., Influence of the hamstring muscles in lumbar spine curvature in sitting.1980; Spine 5(6):525-528.

18. Brittany, L., Hansberger. Evaluating the Relationship between clinical Assessment of Apparent Hamstring tightness: A correlational Analysis. Int J Sports Phys Ther, Apr 2019;14(2):253-263.

19. Jandre, R., F. Macedo., Influence of Hamstring tightness in pelvic, lumbar, and trunk range of motion in low back pain and A-symptomatic volunteers during forwarding bending. Asian spine. 2015; 9(4): 535-540. 